\title{
Dictionnaire de l'autobiographie. écritures de soi de langue française, sous la direction de Françoise
} SIMONET-TENANT

\section{Gabriella Bosco}

\section{(2) OpenEdition}

\section{Journals}

\section{Edizione digitale}

URL: https://journals.openedition.org/studifrancesi/12336

DOI: 10.4000/studifrancesi. 12336

ISSN: 2421-5856

\section{Editore}

Rosenberg \& Sellier

\section{Edizione cartacea}

Data di pubblicazione: 1 avril 2018

Paginazione: 186-187

ISSN: 0039-2944

\section{Notizia bibliografica digitale}

Gabriella Bosco, «Dictionnaire de l'autobiographie. écritures de soi de langue française, sous la direction de Françoise sImonet-tenant», Studi Francesi [Online], 184 (LXII | I) | 2018, online dal 04 juillet 2018, consultato il 18 novembre 2021. URL: http://journals.openedition.org/studifrancesi/12336 ; DOI: https://doi.org/10.4000/studifrancesi. 12336

Questo documento è stato generato automaticamente il 18 novembre 2021.

\section{(c) $(1) \odot$}

Studi Francesi è distribuita con Licenza Creative Commons Attribuzione - Non commerciale - Non opere derivate 4.0 Internazionale. 


\title{
Dictionnaire de l'autobiographie. écritures de soi de langue française, sous la direction de Françoise
} SIMONET-TENANT

\author{
Gabriella Bosco
}

\section{NOTIZIA}

Dictionnaire de l'autobiographie. écritures de soi de langue française, sous la direction de Françoise SIMONET-TENANT, avec la collaboration de Michel Braud, Jean-Louis Jeannelle, Philippe Lejeune et Véronique Montémont, Honoré Champion, Paris, 2017, 845 pp.

1 Ispirato ad analogo strumento per i paesi di lingua inglese - l'Encyclopedia of Life Writing: Autobiographical and Biographical Forms (Londres-Chicago, Fitzroy Dearborn Publishers, 2001) - , e frutto di lunghi anni di lavori da parte di un'équipe composta dai principali specialisti francesi delle scritture autobiografiche, il dizionario pubblicato da Honoré Champion a cura di Françoise SIMONET-TENANT era molto atteso dagli studiosi del genere.

2 In linea per concezione con la serie dei dizionari che da anni l'editore parigino propone (molto interessanti tra quelli usciti, ad esempio, i poderosi volumi dedicati a Diderot, a Marguerite Yourcenar e a Eugène Ionesco), il Dictionnaire de l'autobiographie si presenta, con le sue 457 voci organizzate in base a varie categorie di appartenenza - autori, opere, generi, nozioni tecniche e termini letterari, supporti e strumenti, temi, luoghi di provenienza, epoche e movimenti letterari, strumenti critici - come il tentativo di fare il punto su una riflessione teorica avviata nel 1975 da Philippe Lejeune con il suo Pacte autobiographique, e da allora sviluppatasi intensamente, in forme a volte ipertrofiche e talora incoerenti, a testimonianza comunque di un fortissimo interesse sia da parte degli studiosi che da parte dei lettori nei confronti di quello che è riduttivo chiamare semplicemente un genere, per via delle sue molteplici e sempre nuove incarnazioni. 
3 Il dato di partenza, criticamente discutibile ma che la curatrice assume e fa suo, è quello dell'individuazione, nella seconda metà degli anni Settanta del Novecento, in concomitanza con l'uscita dell'importante saggio di Lejeune, di un ritorno del soggetto nella scrittura narrativa (altra corrente critica sostiene e dimostra con validi argomenti come il soggetto non sia mai sparito dalla scrittura narrativa, neppure nel pieno della sperimentazione neoavanguardistica più spinta, e come semplicemente, attraverso il lavoro teorico e pratico delle neoavanguardie, il soggetto narrativo sia diventato altro rispetto a quello che era prima: di conseguenza, sarebbe abusivo parlare di "ritorno", e bisognerebbe invece prendere atto dell'evoluzione che la nozione di soggetto e il suo statuto hanno ereditato dalla riflessioni sviluppatesi negli anni Sessanta e Settanta). Tra le dichiarazioni d'intenti enunciate nella breve introduzione (pp. 7-11), SimonetTenant evoca dunque la necessità di fare chiarezza rispetto a un campo di ricerche la cui estensione è, scrive, «spesso mal compresa». Questa necessità l'ha indotta ad apporre un sottotitolo, in modo da chiarire che il dizionario si occupa non solo di autobiografia in senso stretto, ma anche di tutte quelle che possono essere definite écritures de soi. La specificazione è molto esplicita, per chi studia questo ambito della letteratura: il dizionario intende esplorare ogni forma di scrittura personale che si possa considerare non finzionale. Ovvero, dato l'intorbidarsi delle linee di confine indotto dal diffondersi mediatico della nozione di autofiction in seguito alla sua introduzione nel lessico teorico e critico oltre che creativo da parte di Serge Doubrovsky (Fils, 1977), lo scopo degli estensori era di descrivere le specificità del campo al di qua di ogni contaminazione di tipo per l'appunto finzionale. Fatta questa doverosa precisazione, la curatrice tiene a sottolineare che il dizionario non vuole però essere in alcun modo polemico e che quindi anche ciò che continua a essere oggetto di dibattito - ad esempio la legittimità di far rientrare nel genere autobiografico singole pratiche di scrittura di difficile catalogazione, schematizzazioni sempre in movimento, cronologie non condivise - viene presentato in quanto tale, senza voler mascherare le divergenze, ma piuttosto nel tentativo di chiarire i termini delle querelles, laddove esse sussistono.

4 Non solo autobiografia insomma, ma l'intera nebulosa delle scritture di sé nelle quali è lecito includere il diario, la corrispondenza, le memorie e le testimonianze, ovvero tutti i testi che hanno per oggetto una realtà extralinguistica vista attraverso il prisma di una soggettività. L'autofiction non è esclusa, e la voce figura nel dizionario, proprio in virtù del fatto che la nozione, in continuo processo di ridefinizione teorica, continua $\mathrm{a}$ non fissarsi in maniera definitiva.

5 Il sottotitolo del dizionario è anche indicativo dell'apertura geografica a tutte le scritture francofone: oltre a quella francese, la svizzera, la belga, la quebecchese, l'africana, l'indio-oceanica, la mediorientale, la caraibica, l'asiatica. Così come lo spessore cronologico è arretrato di molto rispetto alla tradizionale convenzione secondo cui la scrittura autobiografica sarebbe nata per la lingua francese con Rousseau. Come ha dimostrato Foucault, se è vero che con Rousseau la pratica dell'autobiografia assume caratteristiche specifiche, è altresì innegabile che «le souci de soi» risale all'Antichità. Ha poi assunto nei secoli modi e forme variabili, ed è di queste - scrive Françoise Simonet-Tenant - che il dizionario vuole rendere conto.

6 La presa di posizione della curatrice è comunque netta: "segregazionista" e non "integrazionista", per usare le sue stesse definizioni: «Il dizionario s'interessa a tutte le scritture di sé, senza gerarchia, postulando che una scrittura in prima persona non 
finzionale pensi e dica qualcosa d'irriducibile che non può essere trovato altrove». Il che non implica che tutti i 192 collaboratori condividano necessariamente tale assolutezza. Si può così anche trovare nel dizionario la voce «Antiautobiografia»e articoli su autori tradizionalmente considerati lontani dalla scrittura non finzionale.

7 Di grande utilità le bibliografie necessariamente selettive ma proprio per questo spesso molto efficaci dal punto di vista funzionale con cui termina ogni voce. 